PROCEEDINGS OF THE

AMERICAN MATHEMATICAL SOCIETY

Volume 135, Number 8, August 2007, Pages 2649-2659

S 0002-9939(07)08746-1

Article electronically published on March 21, 2007

\title{
RANK OF THE FUNDAMENTAL GROUP OF ANY COMPONENT OF A FUNCTION SPACE
}

\author{
GREGORY LUPTON AND SAMUEL BRUCE SMITH
}

(Communicated by Paul Goerss)

\begin{abstract}
We compute the rank of the fundamental group of any connected component of the space $\operatorname{map}(X, Y)$ for $X$ and $Y$ connected, nilpotent $\mathrm{CW}$ complexes of finite type with $X$ finite. For the component corresponding to a general homotopy class $f: X \rightarrow Y$, we give a formula directly computable from the Sullivan model for $f$. For the component of the constant map, our formula retrieves a known expression for the rank in terms of classical invariants of $X$ and $Y$. When both $X$ and $Y$ are rationally elliptic spaces with positive Euler characteristic, we use our formula to determine the rank of the fundamental group of any component of $\operatorname{map}(X, Y)$ explicitly in terms of the homomorphism induced by $f$ on rational cohomology.
\end{abstract}

\section{INTRODUCTION}

The rank of a finitely generated abelian group $G$ is the cardinality of a basis of the free abelian group $G / T$, where $T$ is the torsion subgroup of $G$. This notion can be extended to finitely generated nilpotent groups $\Gamma$ by means of the central series $\Gamma=\Gamma_{0} \supset \Gamma_{1} \supset \cdots \supset \Gamma_{n}=\{1\}$, where $\Gamma_{n}=\left[\Gamma_{n-1}, \Gamma\right]$. The rank of $\Gamma$ is the sum of the ranks of the finitely generated abelian quotients $\Gamma_{k-1} / \Gamma_{k}$ for $k=1, \ldots, n$.

Given connected spaces $X$ and $Y$, let $\operatorname{map}(X, Y)$ denote the function space of all (not-necessarily based) maps from $X$ to $Y$ with the compact-open topology. Given a map $f: X \rightarrow Y$, write $\operatorname{map}(X, Y ; f)$ for the connected component of $\operatorname{map}(X, Y)$ containing $f$, that is, the space of maps freely homotopic to $f$. Under reasonable hypotheses on $X$ and $Y$, the fundamental groups of the connected components of $\operatorname{map}(X, Y)$ are finitely generated nilpotent groups. To be precise, say a space $X$ is nilpotent if $\pi_{1}(X)$ is a nilpotent group and the action of $\pi_{1}(X)$ on the higher homotopy groups of $X$ is a nilpotent action. If $X$ is a finite $\mathrm{CW}$ complex and $Y$ is a nilpotent $\mathrm{CW}$ complex of finite type, then the components of $\operatorname{map}(X, Y)$ are themselves nilpotent CW complexes of finite type ([11 and [9, Th.II.2.5]). Thus with these hypotheses $\pi_{1}(\operatorname{map}(X, Y ; f))$ is a finitely generated nilpotent group. Our purpose in this paper is to give a formula for the rank of this group expressed in terms of accessible invariants of the map $f: X \rightarrow Y$.

In [10], we have described the higher rational homotopy groups of $\operatorname{map}(X, Y ; f)$. Our description there is in terms of the homology of chain complexes of derivations

Received by the editors February 1, 2006 and, in revised form, April 13, 2006.

2000 Mathematics Subject Classification. Primary 55Q52, 55P62.

Key words and phrases. Function space, fundamental group, nilpotent space, nilpotent group, rank, rational homotopy, minimal models, derivations.

(C)2007 American Mathematical Society Reverts to public domain 28 years from publication 2649 
of Sullivan minimal models. Specifically, for each $n \geq 2$ we constructed a vector space isomorphism

$$
\pi_{n}(\operatorname{map}(X, Y ; f)) \otimes \mathbb{Q} \cong H_{n}\left(\operatorname{Der}\left(\mathcal{M}_{Y}, \mathcal{M}_{X} ; \mathcal{M}_{f}\right)\right),
$$

where $\operatorname{Der}_{*}\left(\mathcal{M}_{Y}, \mathcal{M}_{X} ; \mathcal{M}_{f}\right)$ is a graded vector space of (generalized) algebra derivations constructed directly from a Sullivan model $\mathcal{M}_{f}: \mathcal{M}_{Y} \rightarrow \mathcal{M}_{X}$ of the map $f: X \rightarrow Y$ (see Section 2 below for precise definitions). Now within the framework established there, we may also compute $H_{1}\left(\operatorname{Der}\left(\mathcal{M}_{Y}, \mathcal{M}_{X} ; \mathcal{M}_{f}\right)\right)$, which is a rational homotopy invariant of the map $f$. But the fundamental group $\pi_{1}(\operatorname{map}(X, Y ; f))$ is generally non-abelian, even after rationalization - see Example 1.1 below. So it is evidently not possible to identify the rationalized fundamental group, as a group, with the (abelian) vector space $H_{1}\left(\operatorname{Der}\left(\mathcal{M}_{Y}, \mathcal{M}_{X} ; \mathcal{M}_{f}\right)\right)$. In other words, the isomorphism (11) does not extend to the case $n=1$. Nonetheless, one intuitively expects this latter vector space to be related to the rationalization of the fundamental group. Our main result of this paper makes their relationship explicit:

Theorem 1. Let $X$ and $Y$ be connected, nilpotent $C W$ complexes of finite type with $X$ finite. Let $f: X \rightarrow Y$ be any given map. Then

$$
\operatorname{rank}\left(\pi_{1}(\operatorname{map}(X, Y ; f))\right)=\operatorname{dim}_{\mathbb{Q}}\left(H_{1}\left(\operatorname{Der}\left(\mathcal{M}_{Y}, \mathcal{M}_{X} ; \mathcal{M}_{f}\right)\right)\right) .
$$

By $\operatorname{dim}_{\mathbb{Q}}$ on the right-hand side of the equation we mean the dimension as a rational vector space.

We prove this result in Section 4 As an illustration of the possible applications, in Theorem 4.1 we express $\operatorname{rank}\left(\pi_{1}(\operatorname{map}(X, Y ; f))\right)$ for a map $f: X \rightarrow Y$ between certain formal spaces directly in terms of the map induced by $f$ on rational cohomology. A particular consequence is Example 4.2 let $G$ be a compact, simple Lie group of rank $n \geq 2, T^{n} \subseteq G$ a maximal torus and $f: S^{2} \rightarrow G / T^{n}$ any map. Then $\pi_{1}\left(\operatorname{map}\left(S^{2}, G / T^{n} ; f\right)\right)$ is finite - that is, has rank 0 -if and only if $f$ is essential.

Specializing Theorem 1 to the case in which $f=0$, we obtain the following corollary. (This result may also be deduced from Corollary 1.6 of 3 .) Let $b_{n}(X)$ denote the $n$th Betti number of $X$. If $n \geq 2$ let $\rho_{n}(Y)$ denote the integer $\operatorname{dim}_{\mathbb{Q}}\left(\pi_{n}(Y) \otimes \mathbb{Q}\right)$ sometimes called the $n$th Hurewicz number of $Y$.

Corollary 1. Let $X$ and $Y$ be connected, nilpotent $C W$ complexes of finite type with $X$ a finite complex of dimension $N$. Then

$$
\operatorname{rank}\left(\pi_{1}(\operatorname{map}(X, Y ; 0))\right)=\operatorname{rank}\left(\pi_{1}(Y)\right)+\sum_{n=2}^{N+1} \rho_{n}(Y) \cdot b_{n-1}(X) .
$$

Our results here and in 10 complement and augment those of others. In addition to [7] and [3], we cite [14] in which the author obtains results for the null-component case. Recent results of Buijs and Murillo 4] overlap with, indeed extend some aspects of, our work. We will discuss our methods after a brief, motivating example.

Example 1.1. Let $X=\mathbb{C} P^{2}$ and let $Y$ be the total space of the principal fibration $K(\mathbb{Z}, 5) \rightarrow Y \rightarrow K(\mathbb{Z} \times \mathbb{Z}, 3)$ whose $k$-invariant is $k: K(\mathbb{Z} \times \mathbb{Z}, 3) \rightarrow K(\mathbb{Z}, 6)$, corresponding to the cup-product $x \cup y \in H^{6}(K(\mathbb{Z} \times \mathbb{Z}, 3) ; \mathbb{Z})$. Here, $x$ and $y$ denote the generators in $H^{3}(K(\mathbb{Z} \times \mathbb{Z}, 3) ; \mathbb{Z}) \cong \mathbb{Z} \times \mathbb{Z}$. We now consider the null-component $\operatorname{map}(X, Y ; 0)$. The Sullivan minimal model of $Y$ is $\mathcal{M}_{Y}=\Lambda\left(x_{3}, y_{3}, z_{5} ; d\right)$ with the single non-trivial differential $d\left(z_{5}\right)=x_{3} y_{3}$. Here a subscript on a generator denotes its degree. The rational homology coalgebra of $X$ is the vector space $\left\langle 1, a_{2}, b_{4}\right\rangle$, 
with $a_{2}$ primitive and the coproduct on $b_{4}$ given by $\Delta\left(b_{4}\right)=b_{4} \otimes 1+1 \otimes b_{4}+a_{2} \otimes$ $a_{2}$. Because we are focussing on the null-component, and because $X$ is a formal space, we may readily assemble the Sullivan minimal model of $\operatorname{map}(X, Y ; 0)$ from Theorems 1.9 and 6.1 of [3]. We may write it as $\Lambda\left(\alpha_{1}, \beta_{1}, \gamma_{1}, x_{3}, y_{3}, \eta_{3}, z_{5} ; \delta\right)$ with non-zero differentials $\delta\left(\gamma_{1}\right)=\alpha_{1} \beta_{1}, \delta\left(\eta_{3}\right)=\alpha_{1} x_{3}+\beta_{1} y_{3}$ and $\delta\left(z_{5}\right)=x_{3} y_{3}$. (In the notation of [3], our generators $\alpha_{1}, \beta_{1}$ and $\gamma_{1}$, for instance, would be written as $x_{3} \otimes a_{2}, y_{3} \otimes a_{2}$ and $z_{5} \otimes b_{4}$, respectively, and the formulas for the differentials were obtained via the comments preceding Theorem 1.9 of that paper.) Then the fact that there are 3 degree-one generators implies that $\operatorname{rank}\left(\pi_{1}(\operatorname{map}(X, Y ; 0))\right)=3$ and the non-zero differential $\delta\left(\gamma_{1}\right)=\alpha_{1} \beta_{1}$ implies that the rationalized fundamental group is non-abelian.

This example indicates that, even for relatively simple choices of $X, Y$ and $f$, the rationalization of $\pi_{1}(\operatorname{map}(X, Y ; f))$ can display quite rich structure. Example 1.1 also illustrates the effectiveness of the models of [7] and [3] for concrete computations. As regards our methods here, we must emphasize an important point about these models, since our proof of Theorem 1 does not use them. These models are often quite effective tools for gleaning information about a nullcomponent $\operatorname{map}(X, Y ; 0)$ (see, for example, [14]). But for $f: X \rightarrow Y$ an essential map, these models are generally quite complicated. By focussing purely on the rank of $\pi_{1}(\operatorname{map}(X, Y ; f))$, we are able to give a "closed form" description that proceeds directly from the ordinary Sullivan model of the map $f: X \rightarrow Y$ and is relatively easy to handle in practice.

\section{DeRivations AND FUnCtion SPACE COMPONENTS}

We assume basic familiarity with localization of nilpotent groups and spaces, as discussed in 9. Recall that a nilpotent space $Y$ (respectively, group $G$ ) admits a rationalization, which we denote $e_{Y}: Y \rightarrow Y_{\mathbb{Q}}$ (respectively, $e_{G}: G \rightarrow G_{\mathbb{Q}}$ ). If $G$ is abelian, then $G_{\mathbb{Q}} \cong G \otimes \mathbb{Q}$. If $f: X \rightarrow Y$ is a map, we write $f_{\mathbb{Q}}: X \rightarrow Y_{\mathbb{Q}}$ for the compositon $e_{Y} \circ f$ and likewise for a group homomorphism. Post-composition with a map of spaces $g: Y \rightarrow Z$ induces a map $g_{*}: \operatorname{map}(X, Y ; f) \rightarrow \operatorname{map}(X, Z ; g \circ f)$. By [9, Th.II.3.1], if $X$ and $Y$ satisfy the hypotheses of Theorem 11 then $\operatorname{map}(X, Y ; f)$ is a nilpotent space and the induced map $\left(e_{Y}\right)_{*}: \operatorname{map}(X, Y ; f) \rightarrow \operatorname{map}\left(X, Y_{\mathbb{Q}} ; f_{\mathbb{Q}}\right)$ is a rationalization. Under these hypotheses we have, for all $n \geq 1$,

$$
\pi_{n}(\operatorname{map}(X, Y ; f))_{\mathbb{Q}} \cong \pi_{n}\left(\operatorname{map}\left(X, Y_{\mathbb{Q}} ; f_{\mathbb{Q}}\right)\right) .
$$

We also assume familiarity with rational homotopy theory using the DG (differential graded) algebra minimal models introduced by Sullivan. The basic facts that we rely on are as follows: Each nilpotent space of finite-type $Y$ has a Sullivan minimal model $\left(\mathcal{M}_{Y}, d_{Y}\right)$ in the category of nilpotent DG algebras over $\mathbb{Q}$. This DG algebra is unique up to isomorphism and is of the form $\mathcal{M}_{Y}=\Lambda W$, a free graded commutative algebra generated by a positively graded vector space $W$ of finite type. A map $f: X \rightarrow Y$ of nilpotent, finite-type spaces also has a Sullivan minimal model, which is a DG algebra map $\mathcal{M}_{f}: \mathcal{M}_{Y} \rightarrow \mathcal{M}_{X}$. The Sullivan minimal model is a complete rational homotopy invariant for a space or a map. Our main reference for this material is [5], although that book restricts to the simply connected case. See [2] for a treatment of the non-simply-connected case.

Fix two DG algebras $\left(A, d_{A}\right)$ and $\left(B, d_{B}\right)$ and a DG algebra map $\phi: A \rightarrow B$. Define a $\phi$-derivation to be a linear map $\theta: A \rightarrow B$ that reduces degree by $n$ and 
satisfies the derivation law $\theta(x y)=\theta(x) \phi(y)+(-1)^{n|x|} \phi(x) \theta(y)$. Let $\operatorname{Der}_{n}(A, B ; \phi)$ denote the vector space of $\phi$-derivations of degree $n \geq 0$. Define a linear map $D: \operatorname{Der}_{n}(A, B ; \phi) \rightarrow \operatorname{Der}_{n-1}(A, B ; \phi)$ by $D(\theta)=d_{B} \circ \theta-(-1)^{|\theta|} \theta \circ d_{A}$. A standard check now shows that $D^{2}=0$ and thus $\left(\operatorname{Der}_{*}(A, B ; \phi), D\right)$ is a chain complex. To ease notation, we will suppress the differential and only write the outermost degree in the homology of a DG space. In particular, $H_{n}(\operatorname{Der}(A, B ; \phi))$ will denote the homology in degree $n$ of the chain complex $\left(\operatorname{Der}_{*}(A, B ; \phi), D\right)$.

In [10] we showed that the homology theory of these chain complexes may be used to model the rational homotopy theory of function spaces at the level of (higher) homotopy groups. The link between the two is provided by a map

$$
\Phi_{f}: \pi_{n}(\operatorname{map}(X, Y ; f)) \rightarrow H_{n}\left(\operatorname{Der}\left(\mathcal{M}_{Y}, \mathcal{M}_{X} ; \mathcal{M}_{f}\right)\right),
$$

where $\mathcal{M}_{f}: \mathcal{M}_{Y} \rightarrow \mathcal{M}_{X}$ is any choice of a Sullivan minimal model of $f$. The construction of this map originates with Thom [13] and is as follows. Suppose $\beta \in \pi_{n}(\operatorname{map}(X, Y ; f))$ has adjoint $B: S^{n} \times X \rightarrow Y$. Then $B$ induces a DG algebra map $\mathcal{A}_{B}: \mathcal{M}_{Y} \rightarrow H^{*}\left(S^{n} ; \mathbb{Q}\right) \otimes \mathcal{M}_{X}$ that is of the form

$$
\mathcal{A}_{B}(\chi)=1 \otimes \mathcal{M}_{f}(\chi)+u \otimes \theta_{B}(\chi),
$$

where $u \in H^{n}\left(S^{n} ; \mathbb{Q}\right)$ denotes a generator and $\chi \in \mathcal{M}_{Y}$ is of positive degree. Here $H^{*}\left(S^{n} ; \mathbb{Q}\right)$ is viewed as a DG algebra with zero differential. This expression defines a linear map $\theta_{B}: \mathcal{M}_{Y} \rightarrow \mathcal{M}_{X}$ that reduces the degree by $n$. A standard check shows $\theta_{B}$ is an $\mathcal{M}_{f}$-derivation and a cycle. Set $\Phi_{f}(\beta)=\left[\theta_{B}\right] \in H_{n}\left(\operatorname{Der}\left(\mathcal{M}_{Y}, \mathcal{M}_{X} ; \mathcal{M}_{f}\right)\right)$.

Theorem 2.1. Let $f: X \rightarrow Y$ be a map between connected, nilpotent $C W$ complexes of finite type with $X$ finite. Let $\mathcal{M}_{f}: \mathcal{M}_{Y} \rightarrow \mathcal{M}_{X}$ be any choice of a Sullivan minimal model of $f$. Then for $n \geq 1$ the map

$$
\Phi_{f}: \pi_{n}(\operatorname{map}(X, Y ; f)) \rightarrow H_{n}\left(\operatorname{Der}\left(\mathcal{M}_{Y}, \mathcal{M}_{X} ; \mathcal{M}_{f}\right)\right)
$$

is a well-defined map (of sets). Furthermore, we have:

(a) For $n \geq 2$, $\Phi_{f}$ is a group homomorphism whose rationalization

$$
\Phi_{f_{\mathbb{Q}}}: \pi_{n}\left(\operatorname{map}\left(X, Y_{\mathbb{Q}} ; f_{\mathbb{Q}}\right)\right) \rightarrow H_{n}\left(\operatorname{Der}\left(\mathcal{M}_{Y}, \mathcal{M}_{X} ; \mathcal{M}_{f}\right)\right)
$$

is an isomorphism.

(b) Let $Y$ be an $H$-space and suppose $f$ is null-homotopic. For $n \geq 1, \Phi_{f}$ is a group homomorphism whose rationalization is an isomorphism.

(c) For nilpotent $Y$ of finite type, we have a well-defined surjection of sets

$$
\Phi_{f_{\mathbb{Q}}}: \pi_{1}\left(\operatorname{map}\left(X, Y_{\mathbb{Q}} ; f_{\mathbb{Q}}\right)\right) \rightarrow H_{1}\left(\operatorname{Der}\left(\mathcal{M}_{Y}, \mathcal{M}_{X} ; \mathcal{M}_{f}\right)\right) .
$$

Proof. For $X$ and $Y$ simply connected, part (a) is proven in Theorem 2.1 of [10]. We observe that all parts of that proof remain valid if $X$ and $Y$ are only assumed nilpotent since the same ingredients are available in the nilpotent setting. The argument given in [10] to show that $\Phi_{f}$ is well-defined also remains valid for $X$ and $Y$ nilpotent and $n=1$. We point out, however, that our argument given in 10. to show that $\Phi_{f}$ is a homomorphism breaks down for $n=1$, since at one point it would use the auxiliary space $S^{1} \vee S^{1}$ which is non-nilpotent. For this reason, we give more details for parts (b) and (c)

Part (b) is an easy generalization of the result of Thom [13, Th.2]. We argue that $\Phi_{f}$ is a homomorphism. That the rationalization of $\Phi_{f}$ is both 1-1 and onto then follows from the argument of [10, Th.2.1]. Suppose the multiplication on $Y$ is $m: Y \times Y \rightarrow Y$. We then have an induced multiplication $M: \operatorname{map}(X, Y ; 0) \times$ 
$\operatorname{map}(X, Y ; 0) \rightarrow \operatorname{map}(X, Y ; 0)$ defined by $M(g, h)(x)=m(g(x), h(x))$. Suppose that $\alpha, \beta \in \pi_{n}(\operatorname{map}(X, Y ; f))$ have adjoints $A, B: S^{n} \times X \rightarrow Y$. Then $\alpha+\beta$ may be written as a composition $M \circ(\alpha \times \beta) \circ \Delta: S^{n} \rightarrow \operatorname{map}(X, Y ; 0)$. The adjoint of $\alpha+\beta$, which we write as $C$, is given by $C=m \circ(A \times B) \circ(1 \times T \times 1) \circ \Delta: S^{n} \times X \rightarrow Y$. Here, $T: S^{n} \times X \rightarrow X \times S^{n}$ denotes the switching map. It follows immediately that $\mathcal{A}_{C}(\chi)=u \otimes \theta_{A}(\chi)+u \otimes \theta_{B}(\chi)$ and hence that $\Phi_{f}$ is a homomorphism.

Finally, for part $(\mathrm{c})$, we identify $\pi_{1}(\operatorname{map}(X, Y ; f))_{\mathbb{Q}}$ with $\pi_{1}\left(\operatorname{map}\left(X, Y_{\mathbb{Q}} ; f_{\mathbb{Q}}\right)\right)$ as in (2). Now suppose given $[\theta] \in H_{1}\left(\operatorname{Der}\left(\mathcal{M}_{Y}, \mathcal{M}_{X} ; \mathcal{M}_{f}\right)\right)$. Define $\phi(\chi)=$ $1 \otimes \mathcal{M}_{f}(\chi)+s_{n} \otimes \theta(\chi)$ for $\chi \in \mathcal{M}_{Y}$. Since $\theta$ is an $\mathcal{M}_{f}$-derivation that is a cycle, this defines a DG algebra map $\phi: \mathcal{M}_{Y} \rightarrow H^{*}\left(S^{n} ; \mathbb{Q}\right) \otimes \mathcal{M}_{X} \cong \mathcal{M}_{S^{1}} \otimes \mathcal{M}_{X}$. By the standard correspondence between (DG homotopy classes of) maps of minimal models and (homotopy classes of) maps of rational spaces, this gives a map $F: S^{1} \times$ $X \rightarrow Y_{\mathbb{Q}}$ that satisfies $F \circ i_{2} \sim f_{\mathbb{Q}}: X \rightarrow Y_{\mathbb{Q}}$. We may adjust $F$ into a homotopic map $F^{\prime}: S^{1} \times X \rightarrow Y_{\mathbb{Q}}$ that satisfies $F^{\prime} \circ i_{2}=f_{\mathbb{Q}}: X \rightarrow Y_{\mathbb{Q}}$, so that $F^{\prime}$ has as adjoint a based map $S^{1} \rightarrow \operatorname{map}\left(X, Y_{\mathbb{Q}} ; f_{\mathbb{Q}}\right)$ that represents a homotopy class $\alpha \in \pi_{1}\left(\operatorname{map}\left(X, Y_{\mathbb{Q}} ; f_{\mathbb{Q}}\right)\right)$. Evidently, we have $\Phi_{f_{\mathbb{Q}}}(\alpha)=[\theta]$.

For our proof of Theorem 1, we require certain naturality properties of $\Phi_{f}$. First, suppose given $k: Y \rightarrow Z$, with $X, Y$ and $Z$ connected, nilpotent CW complexes of finite type with $X$ finite. Let $\mathcal{M}_{k}: \mathcal{M}_{Z} \rightarrow \mathcal{M}_{Y}$ be a minimal model. By precomposing with $\mathcal{M}_{k}$, we obtain a map of chain complexes

$$
\left(\mathcal{M}_{k}\right)^{*}: \operatorname{Der}_{*}\left(\mathcal{M}_{Y}, \mathcal{M}_{X} ; \mathcal{M}_{f}\right) \rightarrow \operatorname{Der}_{*}\left(\mathcal{M}_{Z}, \mathcal{M}_{X} ; \mathcal{M}_{f} \circ \mathcal{M}_{k}\right) .
$$

Then for $n \geq 2$, we have a commutative square

$$
\begin{gathered}
\pi_{n}\left(\operatorname{map}\left(X, Y_{\mathbb{Q}} ; f_{\mathbb{Q}}\right)\right) \stackrel{\left(\left(k_{\mathbb{Q}}\right)_{*}\right)_{\sharp}}{\longrightarrow} \pi_{n}\left(\operatorname{map}\left(X, Z_{\mathbb{Q}} ; k_{\mathbb{Q}} \circ f_{\mathbb{Q}}\right)\right) \\
\left.\cong\right|_{\Phi_{k_{\mathbb{Q}} \circ f_{\mathbb{Q}}}} \cong \cong \\
H_{n}\left(\operatorname{Der}\left(\mathcal{M}_{Y}, \mathcal{M}_{X} ; \mathcal{M}_{f}\right)\right) \underset{H\left(\left(\mathcal{M}_{k}\right)^{*}\right)}{\longrightarrow} H_{n}\left(\operatorname{Der}\left(\mathcal{M}_{Z}, \mathcal{M}_{X} ; \mathcal{M}_{f} \circ \mathcal{M}_{k}\right)\right)
\end{gathered}
$$

of group homomorphisms, with isomorphisms where indicated by part (a) of Theorem 2.1. Commutativity of the diagram follows easily from the definitions. Our proof of Theorem 1 uses this commutative diagram of group homomorphisms in the case in which $n=2$.

We also need a naturality property in the case in which $n=1$. Suppose given $f: X \rightarrow Y$ with $X$ and $Y$ connected, nilpotent CW complexes of finite type with $X$ finite, and $k: Y \rightarrow K$ with $K$ a connected $H$-space of finite type. Suppose further that $k \circ f$ is null-homotopic. Then we also have a commutative square

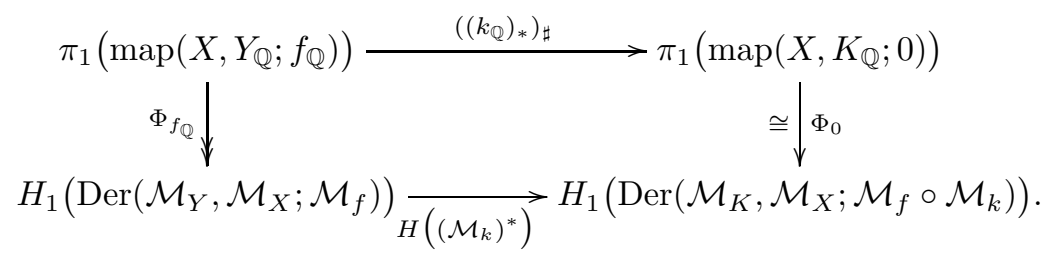

The top horizontal map is a group homomorphism, the right-hand vertical map is a group isomorphism by part (b) of Theorem 2.1, the bottom horizontal map is a group homomorphism and the left-hand vertical map is a surjection of sets. 


\section{Principal fibrations And FunCtion spaces}

Our strategy for proving Theorem 1 is to identify the long exact homology sequence corresponding to a certain principal fibration on function spaces within the framework of chain complexes described above. Recall that a fibration $p: E \rightarrow B$ is principal if $p$ is obtained as a pullback as in the left-hand diagram below:
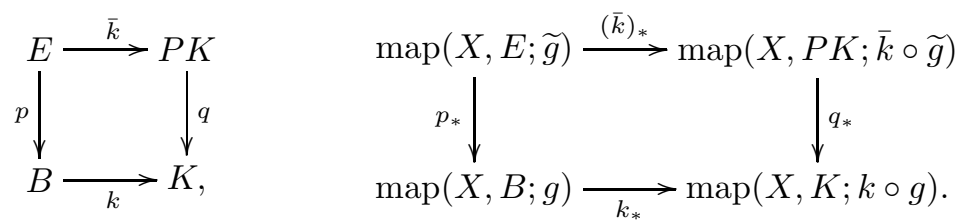

Here, $q: P K \rightarrow K$ is the usual path fibration over some space $K$ and $k: B \rightarrow K$ is some map - usually referred to as the $k$-invariant of the (principal) fibration. The contractibility of $P K$ implies that $E \stackrel{p}{\rightarrow} B \stackrel{k}{\rightarrow} K$ is a fibre sequence.

Lemma 3.1. Suppose given a principal fibration $p: E \rightarrow B$ induced from a $k$ invariant $k: B \rightarrow K$. Let $g: X \rightarrow B$ be a map and $\widetilde{g}: X \rightarrow E$ be a lift of $g$ through p. Then the induced map $p_{*}: \operatorname{map}(X, E ; \widetilde{g}) \rightarrow \operatorname{map}(X, B ; g)$ is a principal fibration induced by the $k$-invariant $k_{*}: \operatorname{map}(X, B ; g) \rightarrow \operatorname{map}(X, K ; 0)$. In particular,

$$
\operatorname{map}(X, E ; \widetilde{g}) \stackrel{p_{*}}{\longrightarrow} \operatorname{map}(X, B ; g) \stackrel{k_{*}}{\longrightarrow} \operatorname{map}(X, K ; 0)
$$

is a fibration sequence.

Proof. Applying map $(X,-)$ to the left-hand pullback diagram of (6) and restricting to appropriate components, we obtain the right-hand diagram of (6), which again is a pullback. Notice that $k \circ p$, and hence $k \circ g$, is null-homotopic. Since $P K$ is contractible, $\operatorname{map}(X, P K)$ is connected and we have $P \operatorname{map}(X, K ; k \circ g) \approx$ $\operatorname{map}(X, P K)$.

We focus on the case $K=K(G, r+1)$ for $r \geq 1$ and describe the rationalization of the long exact homotopy sequence of (7). In this case, we may write the Sullivan minimal model of $E$ as $\mathcal{M}_{E}=\mathcal{M}_{B} \otimes \Lambda \bar{V}$, where $\bar{V}$ is concentrated in degree $r$ and is isomorphic to $G \otimes \mathbb{Q}$. The differential $d_{E}$ restricts to $d_{B}$ on $\mathcal{M}_{B}$ while $d_{E}(\bar{V}) \subseteq \mathcal{M}_{B}$. Furthermore, we may take the minimal model of $p$ to be the DG algebra inclusion $\mathcal{M}_{p}: \mathcal{M}_{B} \rightarrow \mathcal{M}_{E}$. Now suppose given choices of minimal models $\mathcal{M}_{k}: \Lambda V \rightarrow \mathcal{M}_{B}$ and $\mathcal{M}_{g}: \mathcal{M}_{B} \rightarrow \mathcal{M}_{X}$ for $k$ and $g$. Here, $V$ is isomorphic to $G \otimes \mathbb{Q}$ but is concentrated in degree $r+1$ and $\Lambda V$ has trivial differential. We may assume that $\mathcal{M}_{k}(v)=d_{E}(\bar{v})$ for each $v \in V$, where $v \mapsto \bar{v}$ is the obvious degree +1 identification of $V$ with $\bar{V}$. Finally, let $\mathcal{M}_{\tilde{g}}: \mathcal{M}_{E} \rightarrow \mathcal{M}_{X}$ be a minimal model for $\widetilde{g}$ that restricts to $\mathcal{M}_{g}$ on the sub-DG algebra $\mathcal{M}_{B}$.

By pre-composing with $\mathcal{M}_{k}$, as before, we obtain a map of chain complexes

$$
\left(\mathcal{M}_{k}\right)^{*}: \operatorname{Der}_{*}\left(\mathcal{M}_{B}, \mathcal{M}_{X} ; \mathcal{M}_{g}\right) \rightarrow \operatorname{Der}_{*}\left(\Lambda V, \mathcal{M}_{X} ; \mathcal{M}_{g} \circ \mathcal{M}_{k}\right)
$$

Using the mapping cone construction for chain maps (cf. [12, p.166]), we may place the homomorphism induced by $\left(\mathcal{M}_{k}\right)^{*}$ on homology into a long exact sequence of 
the form:

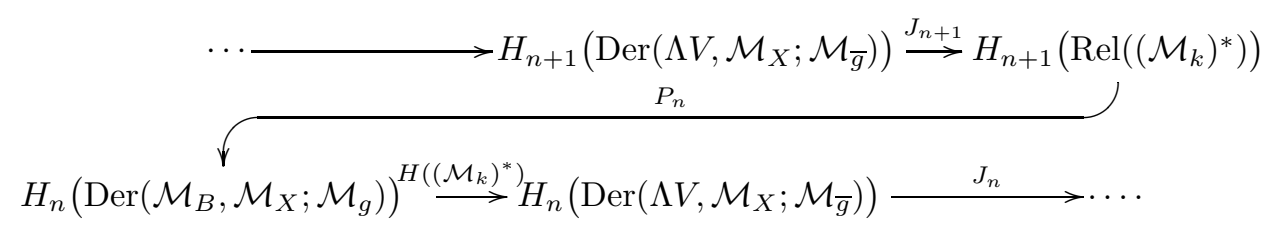

We have written $\bar{g}$ for the composite $g \circ k: X \rightarrow K(G, r+1)$. The "relative" term $\operatorname{Rel}_{*}\left(\left(\mathcal{M}_{k}\right)^{*}\right)$ is the DG vector space with

$$
\operatorname{Rel}_{n}\left(\left(\mathcal{M}_{k}\right)^{*}\right)=\operatorname{Der}_{n-1}\left(\mathcal{M}_{B}, \mathcal{M}_{X} ; \mathcal{M}_{g}\right) \oplus \operatorname{Der}_{n}\left(\Lambda V, \mathcal{M}_{X} ; \mathcal{M}_{\bar{g}}\right)
$$

and differential $\delta: \operatorname{Rel}_{n}\left(\left(\mathcal{M}_{k}\right)^{*}\right) \rightarrow \operatorname{Rel}_{n-1}\left(\left(\mathcal{M}_{k}\right)^{*}\right)$ defined by the rule

$$
\delta(\theta, \varphi)=\left(-D(\theta),\left(\mathcal{M}_{k}\right)^{*}(\theta)+D^{\prime}(\varphi)\right) .
$$

The maps $J_{n}$ and $P_{n}$ are the maps induced on homology by the chain maps given by the obvious inclusions and projections.

Lemma 3.2. Given the above notation, there is a chain isomorphism

$$
\Psi: \operatorname{Rel}_{*}\left(\left(\mathcal{M}_{k}\right)^{*}\right) \rightarrow \operatorname{Der}_{*-1}\left(\mathcal{M}_{E}, \mathcal{M}_{X} ; \mathcal{M}_{\tilde{g}}\right)
$$

of degree -1 . In particular, this induces an isomorphism

$$
H_{2}\left(\operatorname{Rel}_{*}\left(\left(\mathcal{M}_{k}\right)^{*}\right)\right) \stackrel{\cong}{\longrightarrow} H_{1}\left(\operatorname{Der}\left(\mathcal{M}_{E}, \mathcal{M}_{X} ; \mathcal{M}_{\tilde{g}}\right)\right) .
$$

Proof. Given a pair $(\theta, \varphi) \in \operatorname{Rel}_{n}\left(\left(\mathcal{M}_{k}\right)^{*}\right)$ define $\Psi(\theta, \varphi)$ first on $\mathcal{M}_{B}$ by setting it equal to $\theta$. Then, on generators of $\bar{V}$, set $\Psi(\theta, \varphi)(\bar{v})=(-1)^{n} \varphi(v)$ for each generator $v \in V$. Finally, extend to $\mathcal{M}_{B} \otimes \Lambda(\bar{V})$ as an $\mathcal{M}_{\widetilde{g}}$-derivation to obtain an element of $\operatorname{Der}_{n-1}\left(\mathcal{M}_{E}, \mathcal{M}_{X} ; \mathcal{M}_{\widetilde{g}}\right)$. It is easy to check that $\Psi$ is an isomorphism of vector spaces and a chain map.

We will use the following result as the induction step in our proof of Theorem 1. Once again, the hypotheses are indicated in diagram (6).

Proposition 3.3. Suppose given a principal fibration $p: E \rightarrow B$ induced from a $k$ invariant $k: B \rightarrow K(G, r+1)$, some $r \geq 1$. Let $g: X \rightarrow B$ be a map and $\widetilde{g}: X \rightarrow E$ be a lift of $g$ through $p$. Let $X, E$ and $B$ be connected, nilpotent $C W$ complexes of finite type with $X$ finite. Let $G$ be a finitely generated abelian group. If

$$
\operatorname{rank}\left(\pi_{1}(\operatorname{map}(X, B ; g))\right)=\operatorname{dim}_{\mathbb{Q}}\left(H_{1}\left(\operatorname{Der}\left(\mathcal{M}_{B}, \mathcal{M}_{X} ; \mathcal{M}_{g}\right)\right)\right),
$$

then

$$
\operatorname{rank}\left(\pi_{1}(\operatorname{map}(X, E ; \widetilde{g}))\right)=\operatorname{dim}_{\mathbb{Q}}\left(H_{1}\left(\operatorname{Der}\left(\mathcal{M}_{E}, \mathcal{M}_{X} ; \mathcal{M}_{\tilde{g}}\right)\right)\right) .
$$

Proof. The long exact homotopy sequence of (7) displays $\pi_{1}(\operatorname{map}(X, E ; \widetilde{g}))$ as a central extension of the kernel of

$$
\left(k_{*}\right)_{\#}: \pi_{1}(\operatorname{map}(X, B ; g)) \rightarrow \pi_{1}(\operatorname{map}(X, K(G, r+1) ; 0))
$$

by the cokernel of

$$
\left(k_{*}\right)_{\#}: \pi_{2}(\operatorname{map}(X, B ; g)) \rightarrow \pi_{2}(\operatorname{map}(X, K(G, r+1) ; 0)) .
$$

Write the cokernel as $C$. As regards the kernel, set

$$
I=\operatorname{im}\left(k_{*}\right)_{\#}: \pi_{1}(\operatorname{map}(X, B ; g)) \rightarrow \pi_{1}(\operatorname{map}(X, K(G, r+1) ; 0)) .
$$


Then $\operatorname{rank}\left(\operatorname{ker}\left(k_{*}\right)_{\#}\right)=\operatorname{rank}\left(\pi_{1}(\operatorname{map}(X, B ; g))\right)-\operatorname{rank}(I)$, and we have

(8) $\operatorname{rank}\left(\pi_{1}(\operatorname{map}(X, E ; \widetilde{g}))\right)=\operatorname{rank}\left(\pi_{1}(\operatorname{map}(X, B ; g))\right)-\operatorname{rank}(I)+\operatorname{rank}(C)$.

Now we establish a comparable expression in the framework of chain complexes of derivations of minimal models. Choose a minimal model $\mathcal{M}_{k}:(\Lambda V, d=0) \rightarrow \mathcal{M}_{B}$ of the $k$-invariant $k: B \rightarrow K(G, r+1)$. Here, $V$ is a vector space isomorphic to $G \otimes \mathbb{Q}$ concentrated in degree $r+1$. Pre-composition with $\mathcal{M}_{k}$ gives the map of chain complexes $\left(\mathcal{M}_{k}\right)^{*}: \operatorname{Der}_{*}\left(\mathcal{M}_{B}, \mathcal{M}_{X} ; \mathcal{M}_{g}\right) \rightarrow \operatorname{Der}_{*}\left(\Lambda V, \mathcal{M}_{X} ; \mathcal{M}_{g} \circ \mathcal{M}_{k}\right)$ that are featured in Lemma 3.2. The long exact sequence in homology obtained by applying the mapping cone construction to $\left(\mathcal{M}_{k}\right)^{*}$ displays the vector space $H_{2}\left(\operatorname{Rel}\left(\left(\mathcal{M}_{k}\right)^{*}\right)\right)$ as a vector space isomorphic to the direct sum of the cokernel of

$$
H\left(\left(\mathcal{M}_{k}\right)^{*}\right): H_{2}\left(\operatorname{Der}\left(\mathcal{M}_{B}, \mathcal{M}_{X} ; \mathcal{M}_{g}\right)\right) \rightarrow H_{2}\left(\operatorname{Der}\left(\Lambda V, \mathcal{M}_{X} ; \mathcal{M}_{g} \circ \mathcal{M}_{k}\right)\right)
$$

and the kernel of

$$
H\left(\left(\mathcal{M}_{k}\right)^{*}\right): H_{1}\left(\operatorname{Der}\left(\mathcal{M}_{B}, \mathcal{M}_{X} ; \mathcal{M}_{g}\right)\right) \rightarrow H_{1}\left(\operatorname{Der}\left(\Lambda V, \mathcal{M}_{X} ; \mathcal{M}_{g} \circ \mathcal{M}_{k}\right)\right) .
$$

Write this cokernel as $\mathcal{C}$ and set

$$
\mathcal{I}=\operatorname{im} H\left(\left(\mathcal{M}_{k}\right)^{*}\right): H_{1}\left(\operatorname{Der}\left(\mathcal{M}_{B}, \mathcal{M}_{X} ; \mathcal{M}_{g}\right)\right) \rightarrow H_{1}\left(\operatorname{Der}\left(\Lambda V, \mathcal{M}_{X} ; \mathcal{M}_{g} \circ \mathcal{M}_{k}\right)\right)
$$

so that we obtain

$$
\begin{aligned}
\operatorname{dim}_{\mathbb{Q}}\left(H_{2}\left(\operatorname{Rel}\left(\left(\mathcal{M}_{k}\right)^{*}\right)\right)\right)= & \operatorname{dim}_{\mathbb{Q}}\left(H_{1}\left(\operatorname{Der}\left(\mathcal{M}_{B}, \mathcal{M}_{X} ; \mathcal{M}_{g}\right)\right)\right) \\
& -\operatorname{dim}_{\mathbb{Q}}(\mathcal{I})+\operatorname{dim}_{\mathbb{Q}}(\mathcal{C}) .
\end{aligned}
$$

Now, part (a) of Theorem 2.1 and (4) imply that $\operatorname{rank}(C)=\operatorname{dim}_{\mathbb{Q}}(\mathcal{C})$, and parts (b) and (c) of Theorem 2.1 and (5) imply that $\operatorname{rank}(I)=\operatorname{dim}_{\mathbb{Q}}(\mathcal{I})$. Therefore, by (86) and (9), and our hypothesis, we have that

$$
\operatorname{rank}\left(\pi_{1}(\operatorname{map}(X, E ; \widetilde{g}))\right)=\operatorname{dim}_{\mathbb{Q}}\left(H_{2}\left(\operatorname{Rel}\left(\left(\mathcal{M}_{k}\right)^{*}\right)\right)\right) .
$$

The result now follows from Lemma 3.2

\section{Conclusion}

In this section, we prove our main result and its corollary and give a sample application.

Proof of Theorem 1. We obtain by induction the desired formula for an arbitrary Postnikov section $Y^{r}$ of $Y$. That is, we show that

$$
\operatorname{rank}\left(\pi_{1}\left(\operatorname{map}\left(X, Y^{r} ; f^{r}\right)\right)\right)=\operatorname{dim}_{\mathbb{Q}}\left(H_{1}\left(\operatorname{Der}\left(\mathcal{M}_{Y^{r}}, \mathcal{M}_{X} ; \mathcal{M}_{f^{r}}\right)\right)\right)
$$

for any given $r \geq 0$, where $f^{r}: X \rightarrow Y^{r}$ denotes the map induced by $f: X \rightarrow Y$. Since $Y^{0}=\{*\}$, the induction starts trivially.

For the induction step, recall that, since $Y$ is a nilpotent space, by [9, Th.II.2.9] the Postnikov decomposition of $Y$ admits a principal refinement at each stage. That is, $p_{r}: Y^{r} \rightarrow Y^{r-1}$ - the $r$ th stage of the Postnikov decomposition of $Y$-factors into a finite sequence of principal fibrations $\left(c_{r}\right.$ of them, say)

$$
Y^{r}=Y_{c_{r}}^{r} \rightarrow Y_{c_{r}-1}^{r} \rightarrow \cdots \rightarrow Y_{1}^{r} \rightarrow Y_{0}^{r}=Y^{r-1},
$$

each induced from a path-loop fibration by a $k$-invariant of the form $k_{j}^{r}: Y_{j-1}^{r} \rightarrow$ $K\left(G_{j}^{r}, r+1\right)$. Using Proposition 3.3 for each stage of the principal refinement completes the induction.

To complete the proof of Theorem 1 we observe that, by obstruction theory, the $r$-equivalence $p_{r}: Y \rightarrow Y^{r}$ induces an $(r-N)$-equivalence $\left(p_{r}\right)_{*}: \operatorname{map}(X, Y ; f) \rightarrow$ 
$\operatorname{map}\left(X, Y^{r} ; f^{r}\right)$, where $N$ is the dimension of the finite complex $X$. On the derivation complex side, it is straightforward to prove that

$$
\left(\mathcal{M}_{p_{r}}\right)^{*}: \operatorname{Der}_{*}\left(\mathcal{M}_{Y}, \mathcal{M}_{X} ; \mathcal{M}_{f}\right) \rightarrow \operatorname{Der}_{*}\left(\mathcal{M}_{Y^{r}}, \mathcal{M}_{X} ; \mathcal{M}_{f^{r}}\right)
$$

induces an $(r-N)$-homology equivalence. Thus, for $r \geq N+1$, we have

$$
\begin{aligned}
\operatorname{rank}\left(\pi_{1}(\operatorname{map}(X, Y ; f))\right) & =\operatorname{rank}\left(\pi_{1}\left(\operatorname{map}\left(X, Y^{r} ; f^{r}\right)\right)\right) \\
& =\operatorname{dim}_{\mathbb{Q}}\left(H_{1}\left(\operatorname{Der}\left(\mathcal{M}_{Y^{r}}, \mathcal{M}_{X} ; \mathcal{M}_{f^{r}}\right)\right)\right) \\
& =\operatorname{dim}_{\mathbb{Q}}\left(H_{1}\left(\operatorname{Der}\left(\mathcal{M}_{Y}, \mathcal{M}_{X} ; \mathcal{M}_{f}\right)\right)\right) .
\end{aligned}
$$

Proof of Corollary 1. Observe $H_{1}\left(\operatorname{Der}\left(\mathcal{M}_{Y}, \mathcal{M}_{X} ; 0\right)\right) \cong \operatorname{Hom}_{1}\left(Q\left(\mathcal{M}_{Y}\right), H\left(\mathcal{M}_{X}\right)\right)$, where $Q\left(\mathcal{M}_{Y}\right)$ denotes the quotient space of indecomposables of the minimal model of $Y$. The result now follows directly from the fact that $Q_{n}\left(\mathcal{M}_{Y}\right) \cong \pi_{n}(Y) \otimes \mathbb{Q}$ for $n \geq 2$ while $\operatorname{dim}_{\mathbb{Q}}\left(Q_{1}\left(\mathcal{M}_{Y}\right)\right)=\operatorname{rank}\left(\pi_{1}(Y)\right)$.

We conclude with a direct application of Theorem 1. We consider a class of examples for which the rank of the fundamental group of $\operatorname{map}(X, Y ; f)$ is completely determined by the map $H^{*}(f): H^{*}(Y ; \mathbb{Q}) \rightarrow H^{*}(X ; \mathbb{Q})$. We say a simply connected CW complex $Y$ is an $F_{0}$-space if $Y$ is rationally elliptic (rational homology and rational homotopy both finite-dimensional) with positive Euler characteristic. Examples of $F_{0}$-spaces include (products of) even-dimensional spheres, complex projective spaces and, more generally, any homogeneous space $G / H$ with $G$ a compact, connected Lie group and $H$ a closed subgroup of maximal rank. By results of [8], the minimal model for $Y$ takes the form $\mathcal{M}_{Y}=\left(\Lambda V_{0} \otimes \Lambda V_{1}, d_{Y}\right)$ where $V_{0}$ and $V_{1}$ are graded spaces of equal (finite) dimension with $V_{0} \cong \pi_{\text {even }}\left(Y_{\mathbb{Q}}\right)$ evenly graded and $V_{1} \cong \pi_{\text {odd }}\left(Y_{\mathbb{Q}}\right)$ oddly graded. The differential satisfies $d_{Y}\left(V_{0}\right)=0$ while $d_{Y}$ maps $V_{1}$ into the decomposables of $\Lambda V_{0}$. The following extends [6, Th.4.4]:

Theorem 4.1. Let $f: X \rightarrow Y$ be a map between $F_{0}$-spaces where $H^{*}(X ; \mathbb{Q})$ has top degree $2 N$. Let $D_{2}(f)=\operatorname{dim}_{\mathbb{Q}}\left(\operatorname{Der}_{2}\left(H^{*}(Y ; \mathbb{Q}), H^{*}(X ; \mathbb{Q}) ; H^{*}(f)\right)\right)$. Then

$$
\operatorname{rank}\left(\pi_{1}(\operatorname{map}(X, Y ; f))\right)=D_{2}(f)+\sum_{i=1}^{N} \rho_{2 i+1}(Y) \cdot b_{2 i}(X)-\sum_{i=0}^{N} \rho_{2 i+2}(Y) b_{2 i}(X) .
$$

Proof. Write $\rho_{Y}: \Lambda V_{0} \otimes \Lambda V_{1} \rightarrow H^{*}(Y ; \mathbb{Q})$ for the map which sends elements of $V_{0}$ to their corresponding cohomology class and elements of $V_{1}$ to zero. The result follows directly from Theorem 1 and the existence of an exact sequence of the form

$$
\begin{aligned}
0 \rightarrow \operatorname{Der}_{2}\left(H^{*}(Y ; \mathbb{Q}), H^{*}(X ; \mathbb{Q}) ; H^{*}(f)\right) \stackrel{\left(\rho_{Y}\right)^{*}}{\longrightarrow} \operatorname{Der}_{2}\left(\Lambda V_{0}, H^{*}(X ; \mathbb{Q}) ; H^{*}(f) \circ \rho_{Y}\right) \\
\operatorname{Der}_{1}\left(\Lambda V_{1}, H^{*}(X ; \mathbb{Q}) ; 0\right) \stackrel{\mathcal{H}}{\longrightarrow} H_{1}\left(\operatorname{Der}\left(\mathcal{M}_{Y}, \mathcal{M}_{X} ; \mathcal{M}_{f}\right)\right) \rightarrow 0 .
\end{aligned}
$$

Here $\left(\rho_{Y}\right)^{*}$ and $\left(d_{Y}\right)^{*}$ are the maps induced by pre-composition. The map $\mathcal{H}$ is defined as follows. Given $\theta \in \operatorname{Der}_{1}\left(\Lambda V_{1}, H^{*}(X ; \mathbb{Q}) ; 0\right)$, first define a linear map $H(\theta): V \rightarrow \mathcal{M}_{X}$ by setting $H(\theta)\left(V_{0}\right)=\{0\}$ and, on a basis element of $y \in V_{1}$, $H(\theta(y))=P \in \mathcal{M}_{X}$, where $P$ is representative of the class $\theta(y) \in H\left(\mathcal{M}_{X}\right) \cong$ $H^{*}(X ; \mathbb{Q})$. Extend $H(\theta)$ to a linear map of degree -1 , then to an element of $\operatorname{Der}_{1}\left(\mathcal{M}_{Y}, \mathcal{M}_{X} ; \mathcal{M}_{f}\right)$, by the $\mathcal{M}_{f}$-derivation law. The result is a cycle derivation $H(\theta)$. Finally, let $\mathcal{H}(\theta)$ be the homology class of $H(\theta)$. Grivel's proof of [6, Th.4.4] is directly adapted to show $\mathcal{H}$ is well-defined and the sequence is exact. 
As a consequence of Theorem 4.1. we deduce, for instance, the following example.

Example 4.2. Let $G$ be a connected, compact, simple Lie group of rank $n>1$ and $T^{n} \subseteq G$ a maximal torus. Let $f: S^{2} \rightarrow G / T^{n}$ be any given map. We show

$$
\pi_{1}\left(\operatorname{map}\left(S^{2}, G / T^{n} ; f\right)\right) \text { is a finite group if and only if } f \text { is essential. }
$$

The space $G / T^{n}$ is an $F_{0}$-space with rational cohomology generated in degree 2 and we have $b_{2}\left(G / T^{n}\right)=\rho_{2}\left(G / T^{n}\right)=n$. It is well-known that $\rho_{3}(G)=1$, and hence $\rho_{3}\left(G / T^{n}\right)=1$ (see, for instance, p.426 of [1]). Thus by Theorem 4.1, we have $\operatorname{rank}\left(\pi_{1}\left(\operatorname{map}\left(S^{2}, G / T^{n} ; f\right)\right)\right)=D_{2}(f)+1-n$. If $f$ is rationally null-homotopic, then $\operatorname{rank}\left(\pi_{1}\left(\operatorname{map}\left(S^{2}, G / T^{n} ; 0\right)\right)\right)=1$ by Corollary 1. Suppose $f$ is rationally essential. Fix an additive basis $\left\{t_{1}, \ldots, t_{n}\right\}$ for $H^{2}(Y ; \mathbb{Q})$ and suppose, say, $H^{*}(f)\left(t_{i}\right) \neq 0$. Let $\theta_{i} \in \operatorname{Hom}_{2}\left(H^{*}(Y ; \mathbb{Q}), H^{*}(X ; \mathbb{Q})\right)$ be defined on this basis by $\theta_{i}\left(t_{i}\right)=1$ while $\theta_{i}\left(t_{j}\right)=0$ for $j \neq i$. Suppose $\theta_{i}$ extends to an $H^{*}(f)$-derivation. Since the Weyl group of $G$ is a finite reflection group, the cohomology class $t_{1}^{2}+\cdots+t_{n}^{2}$ vanishes in $H^{4}\left(G / T^{n} ; \mathbb{Q}\right)$. However, $\theta_{i}\left(t_{1}^{2}+\cdots+t_{n}^{2}\right)=2 H^{*}(f)\left(t_{i}\right) \theta_{i}\left(t_{i}\right)=2 H^{*}(f)\left(t_{i}\right) \neq 0$. This contradiction implies $D_{2}(f) \leq n-1$ and so $\pi_{1}\left(\operatorname{map}\left(S^{2}, G / T^{n} ; f\right)\right)$ must be a finite group in this case. Finally, by the result of E. Cartan, $\pi_{2}(G)=0$ and so $\pi_{2}\left(G / T^{n}\right)$ is free abelian. Thus $f: S^{2} \rightarrow G / T^{n}$ is essential if and only if it is rationally essential.

\section{REFERENCES}

[1] A. Borel, Topology of Lie groups and characteristic classes, Bull. Amer. Math. Soc. 61 (1955), 397-432. MR0072426 (17:282b)

[2] A. K. Bousfield and V. K. A. M. Gugenheim, On PL de Rham theory and rational homotopy type, Mem. Amer. Math. Soc. 8 (1976), no. 179, ix+94. MR0425956 (54:13906)

[3] E. H. Brown, Jr. and R. H. Szczarba, On the rational homotopy type of function spaces, Trans. Amer. Math. Soc. 349 (1997), no. 12, 4931-4951. MR1407482 (98c:55015)

[4] U. Buijs and A. Murillo, The rational homotopy Lie algebra of function spaces, preprint.

[5] Y. Félix, S. Halperin, and J.-C. Thomas, Rational homotopy theory, Graduate Texts in Mathematics, vol. 205, Springer-Verlag, New York, 2001. MR.1802847 (2002d:55014)

[6] P.-P. Grivel, Algèbres de Lie de dérivations de certaines algèbres pures, J. Pure Appl. Algebra 91 (1994), no. 1-3, 121-135. MR1255925 (95a:55024)

[7] A. Haefliger, Rational homotopy of the space of sections of a nilpotent bundle, Trans. Amer. Math. Soc. 273 (1982), no. 2, 609-620. MR0667163 (84a:55010)

[8] S. Halperin, Finiteness in the minimal models of Sullivan, Trans. Amer. Math. Soc. 230 (1977), 173-199. MR0461508 (57:1493)

[9] P. Hilton, G. Mislin, and J. Roitberg, Localization of nilpotent groups and spaces, NorthHolland Publishing Co., Amsterdam, 1975, North-Holland Mathematics Studies, No. 15. MR0478146 (57:17635)

[10] G. Lupton and S. B. Smith, Rationalized evaluation subgroups of a map I: Sullivan models, derivations, and G-sequences, J. Pure Appl. Algebra, 209 (2007) 159-171.

[11] J. Milnor, On spaces having the homotopy type of CW-complex, Trans. Amer. Math. Soc. 90 (1959), 272-280. MR0100267 (20:6700)

[12] E. H. Spanier, Algebraic topology, Springer-Verlag, New York, 1989. MR0666554 (83i:55001)

[13] R. Thom, L'homologie des espaces fonctionnels, Colloque de topologie algébrique, Louvain, 1956, Georges Thone, Liège, 1957, pp. 29-39. MR0089408 (19:669h)

[14] M. Vigué-Poirrier, Sur l'homotopie rationnelle des espaces fonctionnels, Manuscripta Math. 56 (1986), 177-191. MR0850369 (87h:55009) 
Department of Mathematics, Cleveland State University, Cleveland, Ohio 44115

E-mail address: G.Lupton@csuohio.edu

Department of Mathematics, Saint Joseph's University, Philadelphia, Pennsylvania 19131

E-mail address: smith@sju.edu 